\title{
Advisory Speed for Intelligent Speed Adaptation in Adverse Conditions
}

\author{
Romain Gallen $^{(1)(2)}$, Nicolas Hautière ${ }^{(1)}$ and Sébastien Glaser ${ }^{(2)}$ \\ romain.gallen@lcpc.fr - nicolas.hautiere@lcpc.fr - sebastien.glaser@lcpc.fr \\ (1) Université Paris-Est \\ LEPSiS - LCPC/INRETS \\ 58, boulevard Lefebvre \\ 75015 Paris, FRANCE \\ (2) UniverSud \\ LIVIC - LCPC/INRETS \\ 14 , route de la minière \\ 78000 Versailles, FRANCE
}

\begin{abstract}
In this paper, a novel approach to compute advisory speeds to be used in an adaptive Intelligent Speed Adaptation system (ISA) is proposed. This method is designed to be embedded in the vehicles. It estimates an appropriate speed by fusing in real-time the outputs of ego sensors which detect adverse conditions with roadway characteristics transmitted by distant servers. The method presents two major novelties. First, the $85^{\text {th }}$ percentile of observed speeds $\left(V_{85}\right)$ is estimated along a road, this speed profile is considered as a reference speed practised and practicable in ideal conditions for a lonely vehicle. In adverse conditions, this reference speed is modulated in order to account for lowered friction and lowered visibility distance (top-down approach). Second, this method allows us taking into account the potential seriousness of crashes using a generic scenario of accident. Within this scenario, the difference in speed that should be applied in adverse conditions is estimated so that global injury risk is the same as in ideal conditions.
\end{abstract}

\section{INTRODUCTION}

With 1.27 millions deaths worldwide, road crashes are of major concern. They were the ninth world source of deaths in the world and are expected to be the fifth source in 2030 in developed countries as in developing regions [1]. Indeed, speed is cited as the first factor, it is considered to be the cause in one third of road crashes [2], [3] but it also impacts on the severity of accidents. Many attempts to reduce road fatalities and injuries have been undertaken for fifty years, from speed limitations to seat belt use and drug enforcement. In past decade, automated control of speeds have shown great impact on accidents in England, Netherlands or in France. It is estimated to have helped reduce fatality in France by $25 \%$ between 2003 and 2007 according to [4]. But recent trends showed a stagnation, suggesting that it has reached its full potential for safety benefits.

The development of Advanced Driver Assistance Systems (ADAS) is a very active field of research in the automotive industry. Some widespread systems relying on proprioceptive sensors are integrated in today's cars like the Anti Blocking System (ABS) or the Electronic Stability Program (ESP). Others rely on exteroceptive sensors (LIDAR, RADAR, camera) such as Lane Departure Warning (LDW), Forward Collision Warning (FCW), Traffic Sign Recognition (TSR) or Adaptive Forward Lighting (AFL) systems.

In this context,ADAS focusing on speed such as Intelligent Speed Adaptation (ISA) systems are considered as having high potential for road safety [5]. All types and modes of
ISAs are considered to be highly beneficial for road safety. Firstly, because of the drop in number of crashes due to mean speed decrease, all other things being equal. This has been studied first by Nilsson's power model between speed and accident probability [6], later reviewed in [7]. Other works like [8], [9] confirm that crash-incidence generally decline whenever speed limits have been reduced. Secondly because of the impact of speed on crash severity. Lowering speeds is expected to lead to crashes at lower speeds, thus lowering the seriousness of accidents.

These systems can work in advisory or mandatory modes. Mandatory trials have only been tested in research projects such as [10], [11], [12]. ISAs safety benefits are estimated to be higher for a dynamic ISA in mandatory mode $(-44 \%$ of fatality) compared to dynamic ISA in advisory mode ($9 \%$ of fatality) in case of full penetration of ISA according to [13]. Though recent estimations of safety benefits for ISAs in France [14] have shown lesser potential, ranging from $4 \%$ to $16 \%$ depending on mode and type of road. Current implementations of ISAs compliant with road regulation already exist in advisory mode in many cars with the use of speed limit detection algorithms using cameras such as [15]. New routes are designed according to a back and forth process between the needs and building constraints. Curvatures, slope and superelevation of the road are consistent with the foreseen speed limit. Secondary routes did not benefit of the safety measures of actual building norms. The curvature, the slope and the geometric visibility prevent drivers from driving at the posted speed limit. The posted speed limits are not adaptive enough for precise safety measures. A lonely vehicle should not always consider the posted speed limit as being an advisable speed. Specifically under degraded meteorological conditions when visibility or friction are lowered.

Among recent developments are adaptive dynamic ISAs which aim at being able to cope with various conditions, especially road curvature, lower friction or poor visibility. Finding a safe speed recommandable for a lonely driver in various conditions remains a challenging issue. Curve Overspeed Warning (COW) systems that can adapt speed in curves if speed limit is not suitable also exist such as [16]. The principles for the implementation of an ISA in adverse conditions have been proposed in [17], [18]. These last two 
methods are based on the same safety criterion. The driver must be able to stop the vehicle in the same distance on wet, slippery or dry roads, within the visibility distance.

Using a cooperative scheme between the vehicle's embedded capacity to get real-time information on the environment and road-operators information on roadway characteristics. In the cooperative framework of an adaptive dynamic ISA, this paper presents the computation onboard in real-time of a safe speed along a path. On one side, contextual information estimated in real-time by in-vehicle sensors are used such as fog detection algorithms [19], rainy situations [20], [21] or wet road detection [22]. On the other side, roadway information is used, road operators could provide data such as reference speed, curvature, slope or superelevation in order to expand the electronic horizon before the car. This information is then fused online and the reference speed is modulated depending on environmental and geometric characteristics of the roadway. Our method is original in that the safe speed is not computed from models (bottom-up approach like [18]) but instead a reference speed practised and practicable in good conditions is modulated (top-down approach). The approach for the safety criterion is new and less constraining by taking into account the potential seriousness of a crash using accidents statistics as compared with the "stopping distance" or "zero risk" strategy as used in [17] and [18].

This paper is organized as follows. Section II presents the information received by the car, from the reference speed and its central part in this system to road characteristics and crash statistics integrated in order to calculate potential severity of crashes. In section III the dynamic model of vehicle is presented and in-vehicle estimation of environmental conditions are fused with roadway characteristics. In section IV the output of our ISA on a real path with simulated adverse conditions is shown and analyzed. Finally, section V presents the conclusion and the next steps of our work.

\section{Materials And Methods}

This speed management system is the final output of the French DIVAS project initiated in 2007 [23]. The global scheme of this VII project is presented in Fig. 1. The present article presents the in-vehicle comparison that is done in order to adapt speed to road characteristics (parameters peculiar to the road as curvature, slope, superelevation), vehicle characteristics (parameters such as the presence of ABS or possibly tire quality) and environmental parameters that can only be estimated in real-time (such as wet road or limited visibility conditions such as rain, fog or snow). Road characteristics fall within the competence of road operators, this information is permanent and is transmitted to the car by servers next to the road, the comparison is made onboard in real-time according to the methodology further detailed in section III.

\section{A. Reference Speed}

The $V_{85}$ speed is the $85^{t h}$ percentile of speed distributions. This definition widely used in roadway engineering, either

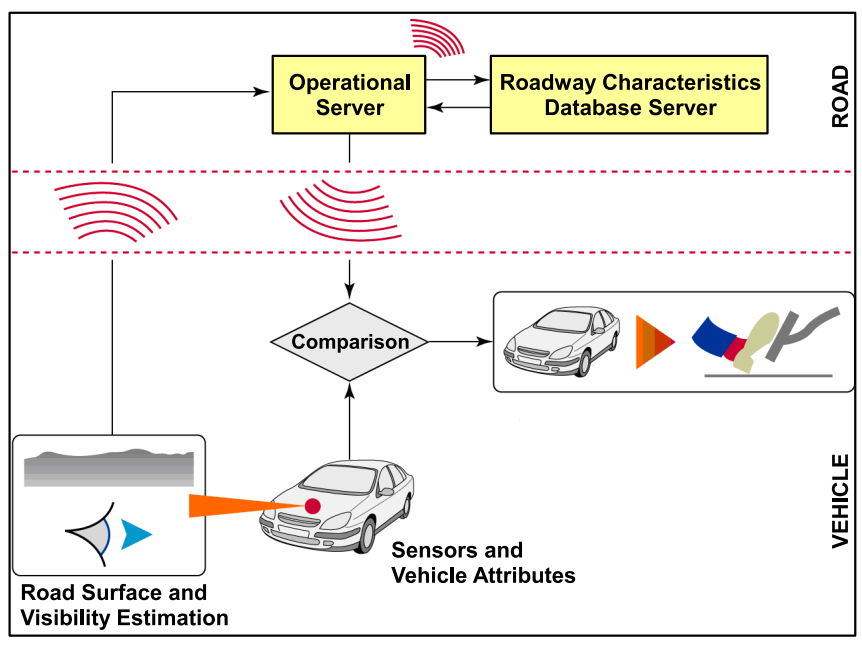

Fig. 1. Layout of Vehicle Infrastructure Integration in DIVAS Project

for design or safety purposes, may have different values depending on the way it is measured. It depends on the nature of vehicles taken into account (light vehicles, trucks etc.). It is also dependent if the vehicle is in traffic or in free-flow (4 to 10 seconds from preceding vehicle) and it is dependent on the moment and the integration time of measure.

Unlike usual measures of $V_{85}$ collected on one location, a continuous profile of $V_{85}$ along a path is used. This profile of reference speed was computed with the methodology presented in [24]. In order to get this profile, several realdriving sessions were made day and night. Profiles where measuring car were constrained by common drivers were suppressed (night courses were easier from this point of view) and the test drivers were asked to follow the path with normal speed and hurried speeding. Fig. 2 presents two extreme profiles observed (the slowest one and the fastest one), the speed limit and the $V_{85}$ finally estimated.

On secondary roads, for a vehicle in free flow conditions with

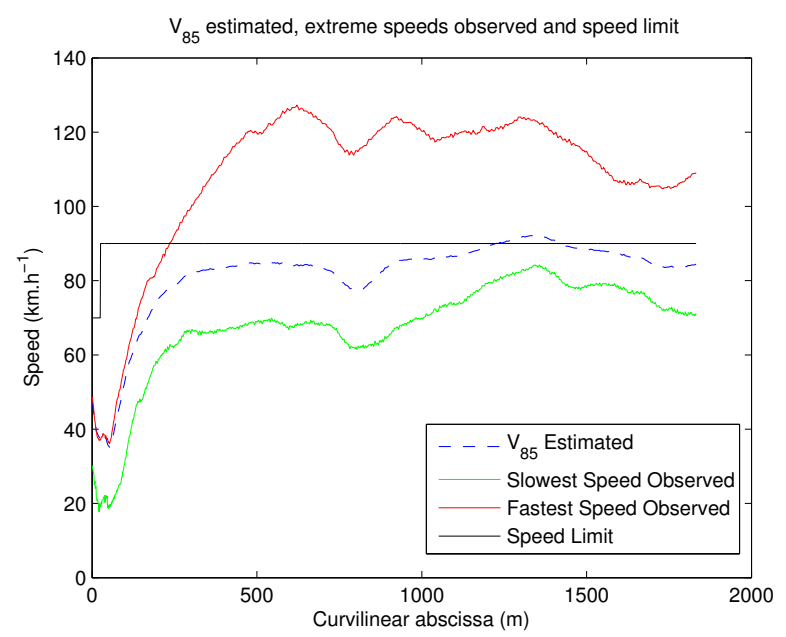

Fig. 2. $\quad V_{85}$ Estimated, extreme speeds observed and speed limit 
ideal meteorological conditions, the $V_{85}$ is a more realistic and acceptable speed to be advised than the legal speed. For legal issues of speed recommandation, compliance of the reference speed with posted speed limits could be necessary. The reference speed to be used can be defined as the $V_{85}$ estimated as long as it is inferior to the posted speed limit.

\section{B. Infrastructure Parameters}

Geometric road characteristics are of major importance concerning the dynamic behavior of vehicles on the road. Many "run off-road" crashes are the consequences of a misunderstanding of the complex interaction between those characteristics and vehicle speed [3]. For example, most drivers don't know the impact of slope on the length of braking or the limits of controllability of their vehicle depending on their speed in regard of the road curvature in turns. A measuring vehicle was driven on this road, it gathers information such as curvature, slope angle and superelevation with a step of $1 \mathrm{~m}$ along the road. These characteristics are considered as static ones, they do not evolve in time. Once acquired this information is available for the whole road. They could be embedded on cars through the use of maps. They could also be transmitted by the infrastructure as it is planed in the VII integration of our project. An electronic horizon of $300 \mathrm{~m}$ is sufficient in order to compute emergency braking profiles according to the methodology detailed in section III.

All the characteristics are not alike. Friction, which is a major characteristic of the road is mainly linked to road roughness, tires nature (slick or engraved) and water height. Our measuring vehicle is able to estimate friction for a standard tire under $1 \mathrm{~mm}$ of water. This characteristic is assumed to be semi-static. The profile of friction can be transmitted to the car as other characteristics of the road. Water height will be measured by roadside units and refined in real-time with camera using algorithms such as [20], [21], [22]. Then, using a model of friction estimation depending on water height, real friction can be estimated at a given time in the vicinity of the car.

\section{Severity Statistics}

Another information that is needed in order to account for potential seriousness is the severity of accidents depending on the speed of a crash and the configuration (frontal collision with a rigid fixed object, with another vehicle or with a stopped vehicle). Many studies have described the link between speed of crashes and severity for the driver or for other vehicle occupants. This severity may depend on driver characteristics such as age, gender or weight [2], [25], on the vehicle safety devices [26], [27], on the direction of collision [28], on the mass ratio in two-vehicle crashes [29] or on the size of cars [30]. These results often show great disparity as some drivers might get seriously injured in crashes at low speeds while some may survive at crashes at very high speeds.

Many different measures linked to speed are used to assess potential severity of crashes. These are Kinetic Energy
Equivalent Speed (KEES or EES), Equivalent Barrier Speed (EBS), Occupant Impact Velocity (OIV) or Acceleration Severity Index (ASI). Some of these measures were designed to relate vehicle kinematics at the instant of crash (derived from post crash observation of vehicle deformation) while other speed related parameters were designed to study potential injury severity. They can be computed from post-crash analysis of vehicle or nowadays from Event Data Recorders (EDR) integrated in cars.OIV and ASI were found by [31] to offer no significant predictive advantage over the simpler delta- $\mathrm{V}(\Delta V)$. Delta- $\mathrm{V}$ is an indication of the acceleration experienced by car occupants while EES assesses the work done in crushing the car structure [32].

According to [33], from analysis of collision data for Britain, Australia and the US, the collisions can all be considered to be frontal impacts for high collision severity levels (i.e. high $\Delta V$ values). The risk for vehicle occupants is estimated by using statistics relating injury severity probability as a function of delta- $\mathrm{V}$ in frontal impacts taken from [34] as shown on Fig. 3.

In order to dispose of continuous cumulative crash severity curves depending on delta- $\mathrm{V}$ given in [34], sigmoid functions were fitted to the data, they are of the form:

$$
P I_{\text {Severity }}(\Delta V)=\frac{a_{\text {Severity }}}{1+e^{-\frac{\Delta V-b_{\text {Severity }}}{c_{\text {Severity }}}}}
$$

where a, b and c are coefficient depending on the severity, which can be either slight, serious or fatal. There are three sets of coefficients, one for each probability of injury (PI) curve such as presented in Tab. I.

\section{METHOD}

Our method is based on a basic scenario of accident. While the case vehicle is driving in free-flow condition, an emergency situation occurs. The driver begins an emergency braking manoeuver and will eventually crash against a rigid fixed obstacle. The cause of the emergency situation is

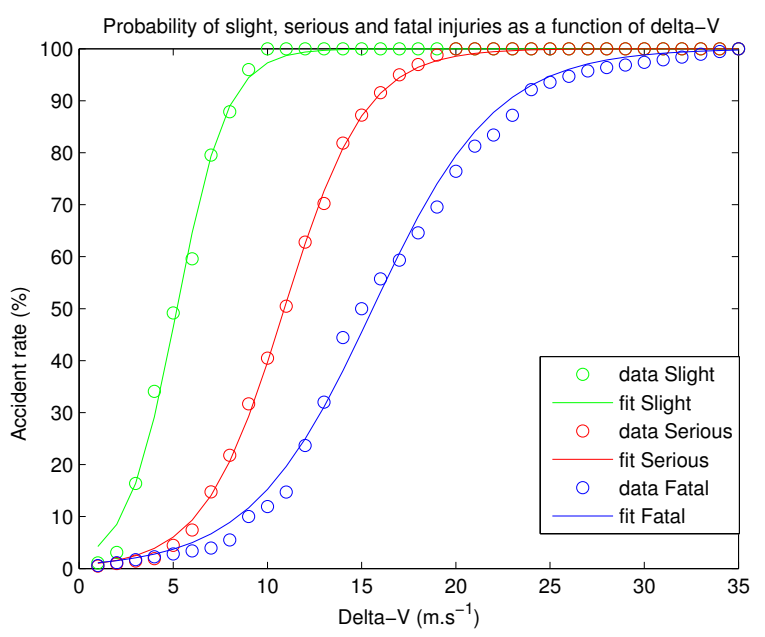

Fig. 3. Cumulative speed curves for drivers in frontal impacts, data from [34] and curve fitting. 
TABLE I

COEFFICIENTS OF FITTING FOR PROBABILITY OF INJURY (PI) CURVES DEPENDING ON SEVERITY AS SHOWN ON FIG. 3

\begin{tabular}{|c||c|c|c|}
\hline Severity & \multicolumn{3}{|c|}{ Coefficients } \\
\hline \hline Slight & $a_{S l}=100$ & $b_{S l}=5.19$ & $c_{S l}=1.34$ \\
Serious & $a_{S e}=100$ & $b_{S e}=10.9$ & $c_{S e}=2.15$ \\
Fatal & $a_{F a}=100$ & $b_{F a}=15.6$ & $c_{F a}=3.26$ \\
\hline
\end{tabular}

not treated (inattention, surprise) but may happen anywhere during the trip. In this scenario, it is considered that there is an equiprobability of hitting the obstacle depending on the distance. The driver may run off-road anytime after the detection of an emergency situation and then hit an obstacle. In this scenario, the driver brakes on the road and hits an obstacle on the road or near the road. As the friction outside the road is assumed to be negligible, the driver hits the obstacle with the same speed that he had when he ran offroad.

The goal of the method is to compare the risk for the driver in adverse conditions with regard to the reference risk (i.e. the risk in good conditions). Two speed profiles during Emergency Braking are computed. One profile is computed with good friction and good visibility conditions and one is computed with adverse conditions detected in real-time (such as rain that may imper friction and/or visibility or such as fog that impacts mainly on visibility).

\section{A. Emergency Braking}

The speed profile during an emergency braking is computed using measured local characteristics of the road such as curvature, slope angle, superelevation and road friction as presented in Sec. II-B. Driver related parameters such as reaction time of the driver and its pressure on the brake pedal are also used in the model of the vehicle dynamic. Finally, vehicle related parameters such as the presence of ABS are used to compute these speed profiles.

After an emergency situation arises, the driver needs some time to be aware of the situation and to start pressuring the brake pedal. During this time of perception and reaction denoted $t_{P R}$, the speed is kept constant and the distance $D_{\text {Reac }}$ covered is:

$$
D_{\text {Reac }}=V_{0} \cdot t_{P R}
$$

with $V_{0}$ the speed in $m . s^{-1}$ and $t_{P R}$ is the perceptionreaction time in seconds. Once the driver has covered this distance with constant speed, the emergency braking takes place with a starting speed equal to $V_{0}$. Braking on straight section and in curves are differentiated when it comes to computing these emergency braking speed profiles.

1) Braking on straight sections: On straight sections all mobilizable friction can be used for the longitudinal braking procedure. The acceleration is expressed as a fraction of $g$, the acceleration of gravity $\left(g=9.81 m . s^{-2}\right)$. The maximum longitudinal acceleration is:

$$
\operatorname{Acc}_{\text {Lon }}(x)=\operatorname{Acc}_{\text {Tot }}(x)=-g(\mu(x)+s(x))
$$

where $x$ is the curvilinear abscissa on the road, $\mu(x) \in[0,1]$ is the friction according abscissa, $s(x)$ is the slope rate according to position (positive for upslope).

The real acceleration used to brake is computed by taking into account the mobilized longitudinal acceleration with the brake pedal pressure and ABS related parameter $\gamma$ with:

$$
A c c_{M o b}(x)=\gamma A c c_{L o n}(x)=-\gamma g(\mu(x)+s(x))
$$

where $\gamma \in[0,1]$ is a parameter linked to the driver's pressure on the brake pedal and the presence of ABS in the vehicle. $\gamma=0.9$ is used when ABS is present in the car and $\gamma=0.7$ when not.

2) Braking in curves: While braking on straight sections allows the driver to mobilize all friction and energy in stopping, braking in curves requires to mobilize part of the friction in order to follow the path. The stopping distance is thus longer than on straight parts of the road. A model of the vehicle dynamics is used in order to account for trajectory keeping. Lateral acceleration depends on the curvature of the road and on the speed. Maximal lateral acceleration is defined as:

$$
A \operatorname{Ac}_{\text {Lat }}(x)=\left|\frac{V(x)^{2}}{R(x)}(\mu(x)+\varphi(x))\right|
$$

where $R(x)$ is the radius of the curve depending on curvilinear abscissa (positive in left turns, negative in right ones) and $\varphi(x)$ is the superelevation angle (positive if the center of lane is higher than the outside). This corresponds to the part of friction mobilized in order to keep the trajectory. Knowing that total deceleration is bounded given a certain amount of friction available so that:

$$
A c c_{\text {Tot }}(x)=\sqrt{A c c_{\text {Lon }}^{2}(x)+A c c_{\text {Lat }}^{2}(x)}
$$

The mobilizable longitudinal acceleration that remains afterwards to brake is:

$\operatorname{Acc}_{\text {Lon }}(x)=-\sqrt{\left(g(\mu(x)-s(x))^{2}-\left(\frac{V(x)^{2}(\mu(x)+\varphi(x))}{R(x)}\right)^{2}\right.}$

Like on straight lines, the real acceleration used to brake is computed by taking into account the mobilized longitudinal acceleration with the brake pedal pressure and ABS related parameter $\gamma$ with:

$$
\operatorname{Acc}_{M o b}(x)=-\gamma \sqrt{\left(g(\mu(x)-s(x))^{2}-\left(\frac{V(x)^{2}(\mu(x)+\varphi(x))}{R(x)}\right)^{2}\right.}
$$

Notice that since $R(x)=\infty$ on straight sections, (8) resumes to (4).

3) Braking Speed Profile: The mobilized longitudinal acceleration during braking at each position either on straight sections or in curves is known with equations (4) and (8). The precise speed profile during braking $V_{B r a k}(x)$ is computed given a step $d x=1 \mathrm{~m}$ with an iterative procedure. For each 


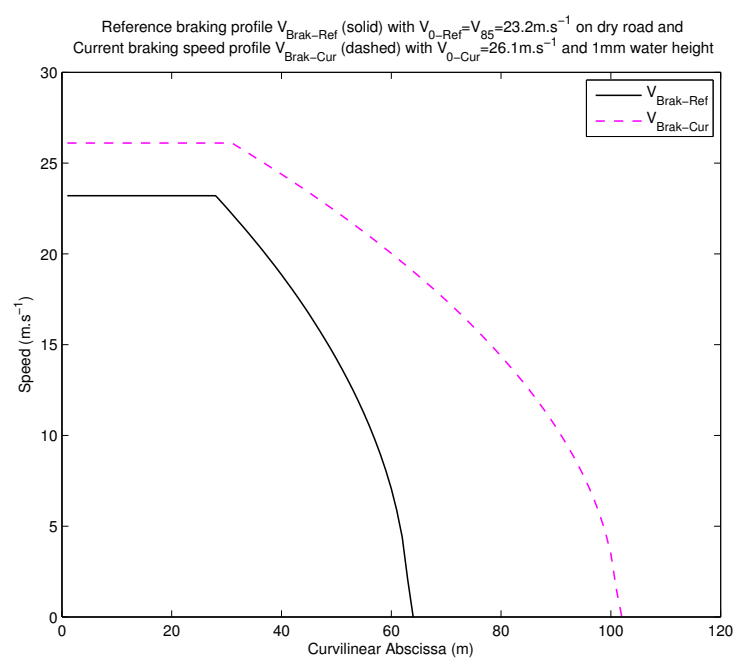

Fig. 4. Emergency braking speed profiles $V_{B r a k}$ at one point on the road in reference condition (dry road) and in current situation (wet road)

position until $V(x)=0$ (i.e. the vehicle has completely stopped) the following equation is solved:

$$
\frac{V_{x+d x}^{2}-V_{x}^{2}}{2 d x}=A c c_{M o b}(x)
$$

Knowing the static characteristics of the road ahead $R(x)$, $s(x), \varphi(x)$. Given fixed parameters linked to vehicle and driver $t_{P R}$ and $\gamma(x)$. For a given position on the road, two different braking speed profiles are computed. One profile for which the braking starts with the real speed of the vehicle $V_{\text {Real }}$ and the estimated semi-static characteristic $\gamma_{\text {Real }}(x)$. One reference profile for which the braking starts with the reference speed $V_{R e f}(x)=V_{85}(x)$ and the reference friction for dry road $\gamma_{\text {Ref }}$.

The braking profiles for the road ahead is computed using (9) for the real conditions and for the reference conditions. The complete profile of speed during an emergency braking is then composed between the initial position and the total stopping distance $\left(D_{S}\right)$ such as shown on Fig. 4. The total stopping distance $D_{S}$ covered from the instant of the emergency situation to complete stop of the vehicle is such that:

$$
D_{S}=D_{\text {Reac }}+D_{\text {Brak }}
$$

The speed profile is the concatenation of a constant speed part of length $D_{\text {Reac }}$ and the braking profile $V_{B r a k}(x)$ of length $D_{B r a k}$ as illustrated on Fig. 4.

Fig. 4 presents two braking speed profiles computed from one position on the road with the real characteristics of the road ahead and a vehicle equipped with ABS. One braking profile corresponds to the profile of the driver as he brakes while driving at $V_{85}$ speed on a dry road. The other profile is the braking profile of the driver in the current situation as he is driving faster than $V_{85}$ on a wet road with $1 \mathrm{~mm}$ water height. Both braking profiles are computed for the same driver and the same vehicle $\left(t_{P R}=1.2 s\right.$ and $\left.\gamma=0.9\right)$.

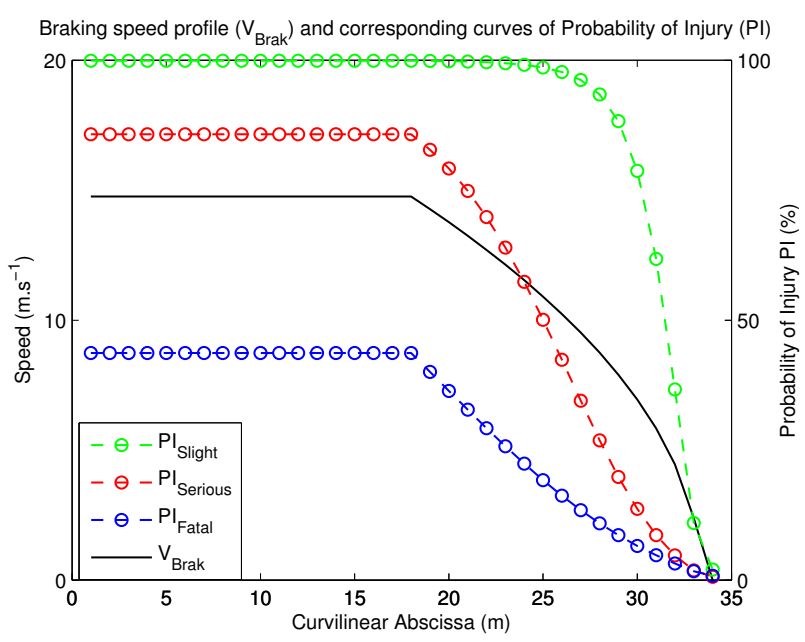

Fig. 5. Braking speed profile $V_{B r a k}$ with parameters $\left(V_{0}=14.8 \mathrm{~m} . \mathrm{s}^{-1}\right.$, $\left.t_{P R}=1.2 s, \mu(x)=\mu_{d r y}(x) \in[0.8,0.9], \gamma=0.9\right)$ and corresponding curves of probability of injury $P I_{S l}, P I_{S e}, P I_{F a}$.

\section{B. Integrating Risk}

1) Crash Scenario: Risk can be defined as the combination of the probability of an incident with its severity. As previously presented in Sec. I, potential seriousness of crashes is taken into account in order to estimate the risk. A basic scenario is defined: the driver has an equiprobability of hitting a static rigid obstacle depending on distance. Considering crashes at different distances, a potential severity measure can be computed knowing the speed at the instant of the crash. As explained in Sec. II-C, delta-V at the instant of the crash is used to infer crash severity potential. As our scenario is based on a crash against a static rigid object, the vehicle is supposed to be completely stopped by the obstacle. In that case the delta- $\mathrm{V}$ for the crash equals the speed at the instant of the crash.

2) Computing Potential Severity: Using the braking profiles $\left(V_{\text {Brak }}\right)$ shown on Fig. 4, and the curves of probability of injury $(P I)$ shown on Fig. 3, for each position until total stopping of the vehicle $D_{S}$ the probability of slight, serious and fatal injury $\left(P I_{S l}, P I_{S e}\right.$ and $\left.P I_{F a}\right)$ are estimated. The different estimations of $P I$ are computed according to the coefficients given in Tab. I with (1). The probability of injury for a crash ahead of the vehicle is:

$$
P I_{\text {Severity }}(x)=P I_{\text {Severity }}\left(V_{\text {Brak }}(x)\right)
$$

Fig. 5 shows how the resulting probability of injury is computed for a given profile of $V_{B r a k}$. Such probability of injury curves can be computed for the three levels of severity at each instant on the road.

3) Existing Approach: Existing approaches like [17], [18] focus on stopping before hitting an obstacle on the road. This strategy of handling risk has three major issues. First of which, an obstacle may fall anywhere on the road or if in an emergency situation, the driver may run off-road in the vicinity and hit an obstacle with high speed. Distant obstacles are not the most dangerous ones. The second issue is that this 


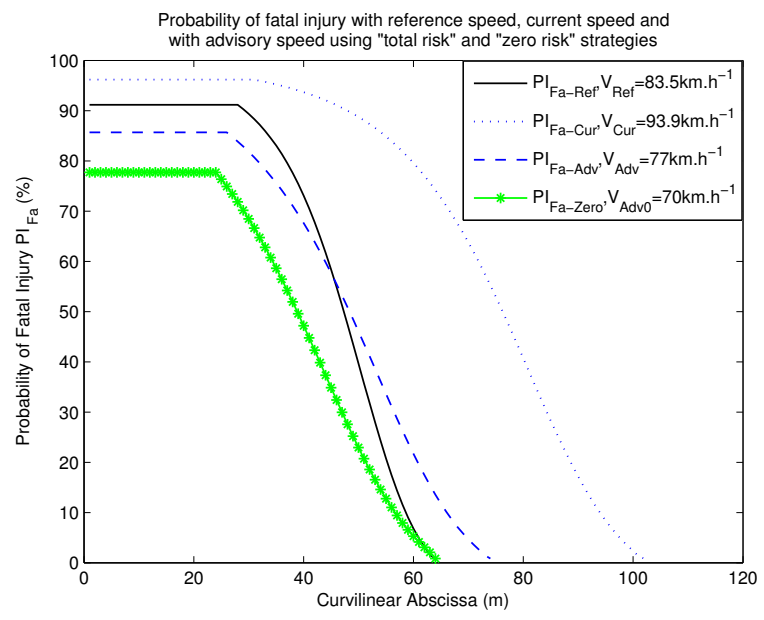

Fig. 6. Probability of injury $P I_{F a}$ for emergency braking.

can be considered as a "zero risk" strategy by trying to avoid any contact with the obstacle while a low speed crash may not be of severe consequences. Thirdly because too cautious strategies can lead to advisory speeds much lower than legal speed, thus lowering credibility of the advise and impairing the final efficiency of an ISA.

Using "zero risk" strategy in a situation like presented on Fig. 4 would lead to lowering the initial speed on wet road such that the stopping distance $D_{S-\text { Real }}$ is the same as on dry road $D_{S-R e f}$.

4) Equivalent Total Risk: Our approach consists in lowering initial speed under adverse conditions of friction or visibility in such a way that total probability of injury in adverse conditions is equal to total probability of injury in reference conditions. This strategy is illustrated in Fig. 6 in one point of the road. A dichotomic procedure is then used to search for an initial speed in adverse conditions such that total probability of fatal injury during braking is equivalent to the total reference probability of fatal injury.

Fig. 6 presents the fatal $P I$ curve in reference conditions for a driver at reference speed $V_{R e f}=83.5 \mathrm{~km} . \mathrm{h}^{-1}$, on a dry road with $\mu_{R e f} \in[0.83,0.88]$ on this section of the road (plain line). At first, the current $P I$ (dotted line) for the current conditions is estimated with $V_{C u r}=93.9 \mathrm{~km} \cdot \mathrm{h}^{-1}$ on a wet road with $1 \mathrm{~mm}$ water height $\left(\mu_{C u r} \in[0.47,0.51]\right)$. If the total current $P I_{F a-C u r}$ is superior to reference $P I_{F a-R e f}$, an advisory speed $V_{A d v}=77 \mathrm{~km} . \mathrm{h}^{-1}$ is computed such as total $P I_{F a-A d v}$ with advisory speed (dashed line) equals total reference $P I_{F a-R e f}$. Notice that with our advisory speed, the stopping distance is slightly longer than in reference conditions ( $74 m$ versus $64 m$ ).

The probability of fatal injury using strategies such as proposed in [17], [18], denoted "zero risk" strategy (star line), is also presented for comparison. Using this method leads to an advisory speed such as stopping distance equals reference stopping distance $(64 \mathrm{~m})$. It was expected that such strategy would lead to the lower advisory speed $\left(V_{A d v 0}=\right.$ $\left.70 k m . h^{-1}\right)$.

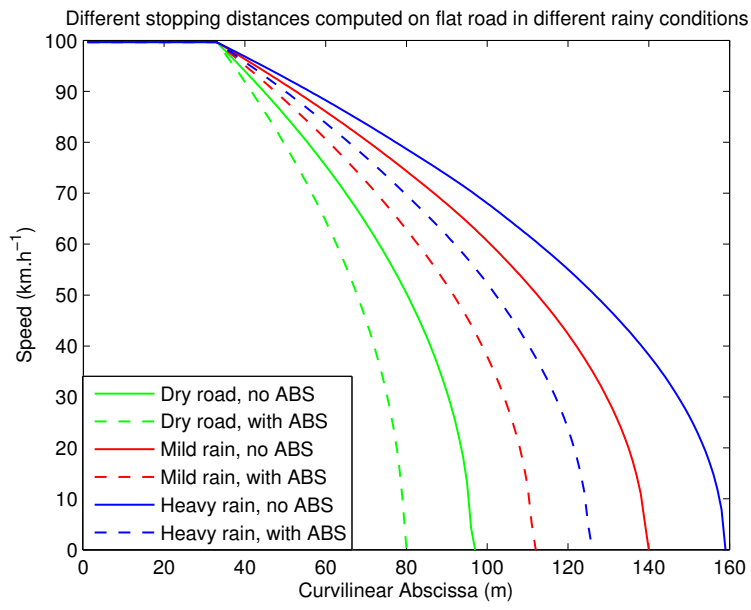

Fig. 7. Emergency braking speed profiles under various rainy conditions.

At any given point along the road, the risk in current situation and the risk in reference situation are compared. From this comparison a lower speed can be proposed in order to limit the current risk to the same level as the reference risk. In the next section the whole profiles of such advised speeds along the road are presented depending on different adverse conditions.

\section{RESUlts}

Fig. 6 shows the result of the methodology that leads to an advisory speed with our "total risk" strategy. Using this methodology allows us proposing at a given position on the road three different advisory speeds depending on the potential severity taken into account. Accounting for "slight injuries" leads to a more cautious speed than accounting for "fatal injuries". In the following sections, the profiles of advisory speeds depending on different adverse conditions along the whole road are presented. The advisory speeds computed with our method will be compared to the reference speed on dry road (i.e. the $V_{85}$ speed in good weather) and to the advisory speed computed with an existing method such as computed with "zero risk" method.

\section{A. Degraded friction in rainy weather}

Adverse conditions of friction are a major issue for road safety as it can lower friction on the road. National statistics of crashes [4] show an increase in the number of accidents under rainy conditions. Though drivers are often confronted to those conditions they are not aware of the impact of different rainy conditions on trajectory keeping and length of braking. Fig. 7 illustrates different speed profiles computed for the same driver on a car with and without ABS in increasing rainy conditions. The total stopping distance is almost doubled depending on the presence of ABS and the intensity of rain. It is possible to compute different braking profiles under various rainy conditions, it is also possible to compose these braking profiles with the curves of probability 


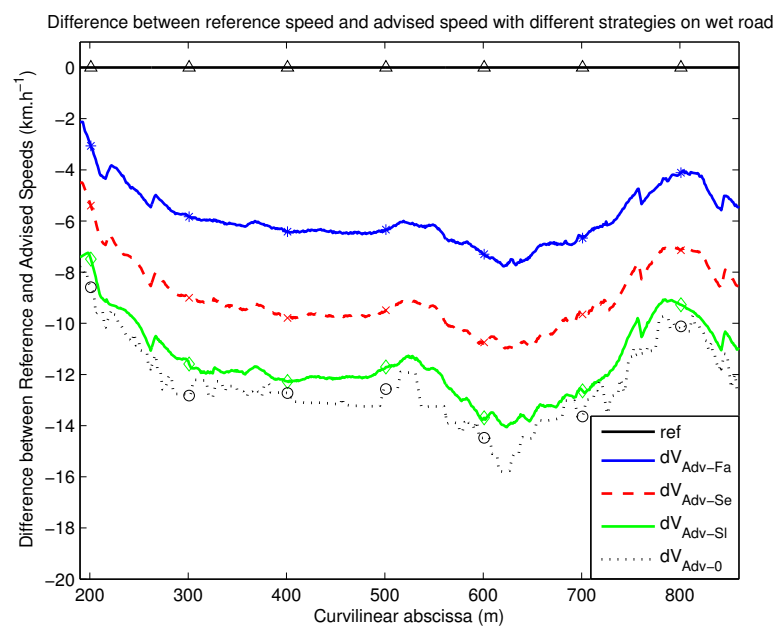

Fig. 8. Difference of speed proposed between reference speed and advisory speeds computed with different safety criterions on wet road.

of injury in order to propose an advisory speed along the road as shown in Fig. 8.

Notice that the advisory speed taking into account slight severity injuries is very close to the advisory speed proposed with the "zero risk" strategy proposed by [17], [18]. This was expected because slight injury curves are very sharp for low speeds and saturated over $10 \mathrm{~ms}^{-1}$ (See Fig. 3). Which means that only the end of braking speed profiles are used to compensate for "total risk" surface strategy. The "zero risk" strategy can be assimilated to "total risk" strategy with a severity curve equal to $100 \%$ from $\Delta V=1 \mathrm{~m} . \mathrm{s}^{-1}$ ( $100 \%$ probability of severity whatever the speed of the crash, meaning the crash should be absolutely avoided).

\section{B. Degraded visibility in fog}

Fog essentially impairs visibility on the road. National statistics of crashes [4] show that it increases the mean severity of crashes, meaning drivers collide with obstacle at higher speeds. Potential severity calculations are adapted by considering that the probability of injury is constant after the distance of visibility as shown in Fig. 9. The probability of injury depending on severity is set constant from the limit of visibility until the total stopping distance. This has no impact if braking speed profile has a total stopping distance inferior to the visibility distance.

As for rainy situations advisory speed along the whole road is computed in the presence of fog limiting visual range to $100 \mathrm{~m}$ as shown in Fig. 10

\section{CONClusion And Future Works}

This paper presents a new approach to compute an advisory speed to be used in an ISA. By using measured characteristics of the road, precise speed profiles in emergency braking are computed. The system proposed is able to deal with multiple adverse conditions that impair friction and visibility such as rain, fog or both simultaneously. Vehicle or driver related parameters such as the presence of ABS, the

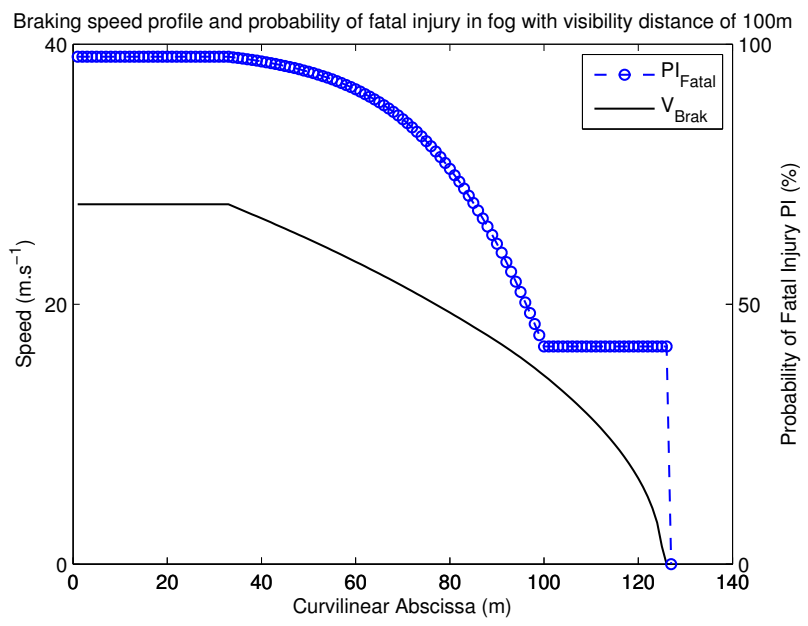

Fig. 9. Braking speed profile and probability of fatal injury in fog with $100 \mathrm{~m}$ of visibility distance.

brake pedal pressure or the perception-reaction time can be used in our model of the vehicle dynamic.

The novelty of this approach also relies on the use of a reference speed considered as safe in reference conditions. This speed is modulated depending on adverse conditions using potential severity criterions. Our advisory speed lies between this safe speed and the speed computed using previous works with a very cautious strategy based on the stopping distance.

In the end three different advisory speeds are computed, these could be qualified as very cautious, cautious and normal advises. When a speed is used in an ISA, it should be kept in mind that greater differences with legal speed or current speed may have less impact and that driver could be tempted not to follow the advise. Over cautious strategies can lead to less efficiency of an ISA as shown in [35].

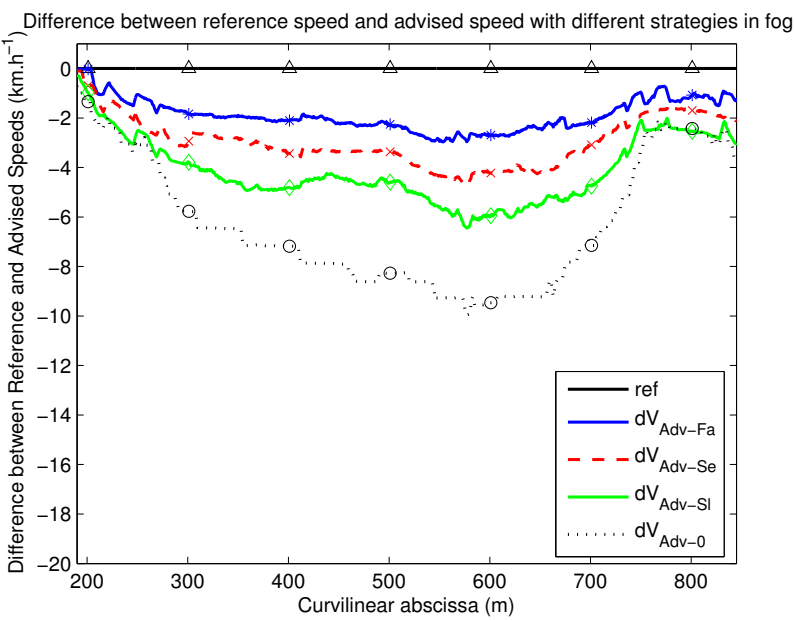

Fig. 10. Difference of speed between reference speed and advisory speeds with different safety criterions in fog with $90 \mathrm{~m}$ of visibility distance. 
The method will further be developed by using other statistics of crashes such as the ratio of "hit rigid fixed obstacles", "hit cars stopped" and "hit facing driving cars" in conjunction with adapted curves of probability of injury corresponding to these scenarios.

We have to choose a strategy for setting parameters such as perception-reaction time or brake pedal pressure. At the present time, these parameters are fixed considering that $t_{P R}=1.2 \mathrm{~s}$ corresponds to a good driver, or more carefully $t_{P R}=2 s$ as it is the $95^{t h}$ percentile of perception reaction time of drivers. Using precise information on a specific driver could lead to advised speeds more adapted to the driver.

We plan on integrating our computation method in a real experiment in France during October 2010.

\section{ACKNOWLEDGMENTS}

This work was supported by the French ANR DIVAS Project. We would like to thank CETE Normandie-Centre for providing us with speed profiles along the road and CETE Lyon for providing us with measured characteristics of the roads.

\section{REFERENCES}

[1] WHO, "The global burden of disease : 2004 update," tech. rep., World Health Organization, Geneva, Switzerland, 2008.

[2] NHTSA, "Traffic safety facts 2008," tech. rep., National Highway Traffic Safety Administration, 2008.

[3] S. McLaughlin, J. Hankey, S. Klauer, and T. Dingus, "Contributing factors to run-off-road crashes and near-crashes," Report NPO-113, NHTSA, USA, 2009.

[4] ONISR, "The major data on accidentology," tech. rep., National road safety observatory, France, 2007.

[5] T.R. Centre, "Speed management," tech. rep., OECD/European Conference of Ministers of Transport, 2006.

[6] G. Nilsson, "The effect of speed limits on traffic accidnets in sweden," Proc. Int. Symp. on the Effects of Speed Limits on Traffic Accidents and Transport Energy Use, Organization for Economic Cooperation and Development, 1981.

[7] R. Elvik, "Speed and road accidents: an evaluation of the power model," tech. rep., Institute of Transports Economics, 2004.

[8] D. Finch, P. Kompfner, C. Lockwood, and G. Maycock, "Speed, speed limits and crashes," Tech. Rep. 58, TRL, Crowthorne, United Kingdom, 1994.

[9] C. Kloeden, G. Ponte, and A. McLean, "Travelling speed and the risk of crash involvement on rural roads," Tech. Rep. CR-204, Australian Transport Safety Bureau, 2001.

[10] O. Carsten and F. Tate, "External vehicle speed control," final report: integration, ITS, Univ. of Leeds, Leeds, 2000.

[11] J. Ehrlich, F. Saad, S. Lassarre, and S. Romon, "Assessment of "lavia" speed adaptation systems: experimental design and initial results on system use and speed behaviour," Proc. of 13th ITS World Congress, United Kingdom, 2006.

[12] A. Varhelyi and T. Makinen, "The effects of in-car speed limiters : Field studies," Transportation Research Part C: Emerging Technologies, vol.9(3), pp.191-211, 2001.

[13] O. Carsten, F. Lai, K. Chorlton, P. Goodman, D. Carslaw, and S. Hess, "Speed limit adherence and its effect on road safety and climate change," tech. rep., University of Leeds - Institute for Transport Studies, october 2008.

[14] R. Driscoll, Y. Page, S. Lassarre, and J. Ehrlich, "Lavia - an evaluation of the potential safety benefits of the french intelligent speed adaptation project," Annu. Proc. Assoc. Adv. Automot. Med., vol.51, pp.485-505, 2007.

[15] N. Barnes, A. Zelinsky, and L. Fletcher, "Real-time speed sign detection using the radial symmetry detector," IEEE Trans. Intell. Transp. Syst., vol.9(2), pp.322-332, 2008.

[16] C. Sentouh, S. Glaser, and S. Mammar, "Advanced vehicleinfrastructure-driver speed profile for road departure accident prevention," Vehicle System Dynamics, vol.44, pp.612-623, 2006.
[17] A. Varhelyi, "Dynamic speed adaptation in adverse conditions -a system proposal-," IATSS Res., vol.26(2), pp.52-59, 2002.

[18] F. Jimenez, F. Aparicio, and J. Paez, "Evaluation of in-vehicle dynamic speed assistance in spain: algorithm and driver behaviour," IET Intell. Transp. Syst., vol.2(2), pp.132-142, 2008.

[19] N. Hautière, J.P. Tarel, J. Lavenant, and D. Aubert, "Automatic fog detection and estimation of visibility distance through use of an onboard camera," Machine Vision and Applications Journal, vol.17(1), pp.8-20, 2006.

[20] S. Gormer, A. Kummert, S.B. Park, and P. Egbert, "Vision-based rain sensing with an in-vehicle camera," Proc. IEEE Intelligent Vehicles Symposium (IV'09), 2009.

[21] J. Halimeh and M. Roser, "Raindrop detection on car windshields using geometric-photometric environment construction and intensitybased correlation," Proc. IEEE Intelligent Vehicles Symposium (IV'09), 2009.

[22] T. Teshima, H. Saito, M. Shimizu, and A. Taguchi, "Classification of wet/dry area based on the mahalanobis distance of feature from time space image analysis," Proc. IAPR on Machine Vision Applications, 2009.

[23] N. Hautière and P. Lepert, "Dialogue between infrastructure and vehicles to improve road safety: The divas approach," Transport Research Arena (TRA'08), Ljubljana, Slovenia, 2008.

[24] G. Louah, "The accuracy of a speed profile estimation method combining continuous and spot speed measurements," 13th Int. Conf. Road Safety on Four Continents, Warsaw, Poland, 2005.

[25] E. Romano, T. Kelley-Baker, and R. Voas, "Female involvement in fatal crashes: Increasingly riskier or increasingly exposed?," Accident Analysis and Prevention, vol.40, pp.1781-1788, 2008.

[26] L. Evans and P. Gerrish, "Antilock brakes and risk of front and rear impact in two-vehicle crashes," Accident Analysis and Prevention, vol.28(3), pp.315-323, 1996.

[27] D. Gabauer and H. Gabler, "Comparison of roadside crash injury metrics using event data recorders," Accident Analysis and Prevention, vol.40, pp.548-558, 2008.

[28] D. Buzeman, D. Viano, and P. Lovsund, "Injury probability and risk in frontal crashes: Effects of sorting techniques on priorities for offset testing," Accident Analysis and Prevention, vol.30(5), pp.583-595, 1998.

[29] E. Miltner and H. Salwender, "Influencing factors on the injury severity of restrained front seat occupants in car-to-car head-on collisions," Accident Analysis and Prevention, vol.27(2), pp.143-150, 1995.

[30] S. Acierno, R. Kaufmanc, F. Rivara, and D. Grossman, "Vehicle mismatch: injury patterns and severity," Accident Analysis and Prevention, vol.36, pp.761-772, 2004.

[31] D. Gabauer and H. Gabler, "Comparison of the delta-v and occupant impact velocity crash severity metrics using event data recorders," Proceedings of the 50th Annual Association for the Advancement of Automotive Medicine Conference, Chicago, IL, USA, 2006.

[32] R. Ross, J. Lenard, B. Hurley, P. Thomas, D. Otte, and G. Vallet, "Crash severity calculations - theory and practice." STandardisation of Accident and Injury Registration Systems (STAIRS), 1998.

[33] D. Wood, N. Veyrat, C. Simms, and C. Glynn, "Limits for survivability in frontal collisions: Theory and real-life data combined," Accident Analysis and Prevention, vol.39, pp.679-687, 2007.

[34] D. Richards and C. Cuerden, "The relationship between speed and car driver injury severity," tech. rep., Transport Research Laboratory, London, UK, 2009.

[35] J. Blum and A. Eskandarian, "Managing effectiveness and acceptability in intelligent speed adaptation systems," Proc. IEEE Intelligent Transportation Systems Conference (ITSC'06), Toronto, Canada, 2006. 